\title{
Lower bounds for relative class numbers of imaginary abelian number fields and CM-fields
}

\author{
by \\ StÉphane R. Louboutin (Marseille)
}

\section{INTRODUCTION}

We give explicit upper and lower bounds for relative class numbers of imaginary abelian number fields and of nonabelian CM-fields $K$. We then use them to obtain Brauer-Siegel type results for relative class numbers of CM-fields. The main feature of this paper is a new method which enables us to easily deal with the case where $K$ contains an imaginary quadratic subfield.

We will make use of the ideas introduced in [Lou04] to obtain in Theorem 1 upper bounds on $|L(1, \chi)|$ for even and odd primitive Dirichlet characters $\chi$, whereas our previous ideas (developed in [Lou98b] and [Lou01]) failed to embrace the case of odd characters. These bounds depend on whether $L(s, \chi)$ has or does not have a real zero $\beta$ in the range $0<\beta<1$. They will then enable us to obtain in Theorems 18, 28 and 31 bounds for relative class numbers $h_{K}^{-}$of CM-fields $K$, especially in the case that $K$ contains an imaginary quadratic subfield $L$. Apart from the proof of Lemma 17, which can be found in [Lou03], this paper provides the reader with a self-contained exposition of how one can obtain (as in Corollaries 20,21, 23 and 25 where several footnotes clearly show that our approach is more efficient than the ones formerly developed by various authors) good enough explicit lower bounds for relative class numbers to enable him to solve various class number problems for CM-fields or to simplify the existing proofs (e.g., see [CK98], [CK00a], [CK00b], [Lou95], [Lou97],[Lou98a], [Lou99], [MM] and [Yam]). Whereas almost all the papers in the literature dealing with explicit lower bounds for relative class numbers of CM-fields (or values at $s=1$ of $L$-functions)

2000 Mathematics Subject Classification: Primary 11M06, 11M20, 11R20, 11R29, $11 \mathrm{R} 42$.

Key words and phrases: $L$-function, relative class number, imaginary abelian number field, CM-field. 
use infinite products of Dedekind zeta functions (e.g., see [Bes], [HHRW], [Lou92], [LPP] and [Sta]), our approach is different and stems from integral representations (used to proved functional equations). We finally refer the reader to $[\mathrm{AD}]$ and $[\mathrm{LO}]$ for conditional (i.e. only valid under the assumption of GRH) lower bounds on the exponents of the ideal class groups of CM-fields.

\section{UPPER BOUNDS FOR $|L(1, \chi)|$}

Throughout this paper, we let $\gamma=0.577215 \ldots$ denote Euler's constant and set

$$
\left\{\begin{array}{l}
\kappa_{0}:=2+\gamma-\log (4 \pi)=0.046191 \ldots \\
\kappa_{1}:=2+\gamma-\log \pi=1.432485 \ldots
\end{array}\right.
$$

In this section we introduce a new method for proving at one stroke the following result:

Theorem 1. Let $\chi$ be a primitive Dirichlet character modulo $f>1$.

(i) We have

$$
|L(1, \chi)| \leq \frac{1}{2}\left(\log f+\kappa_{\chi}\right) \quad \text { where } \quad \kappa_{\chi}:= \begin{cases}\kappa_{0} & \text { if } \chi \text { is even }, \\ \kappa_{1} & \text { if } \chi \text { is odd } .\end{cases}
$$

(ii) Assume that $\chi$ is quadratic. Then $0<\beta<1$ and $L(\beta, \chi)=0$ imply

$$
0<L(1, \chi) \leq \frac{1-\beta}{8} \log ^{2} f
$$

REMARKs 2. (i) O. Ramaré proved in [Ram] that (2) still holds true with the slightly better constants $\kappa_{0}=0$ and $\kappa_{1}=5-2 \log 6=1.416481 \ldots$

(ii) See [Lou98b, Corollaire 7B] for another proof of (3) in the case that $\chi$ is even and quadratic, [Lou01, Theorem 7] for the case of $\chi$ even but not necessarily quadratic, and Theorem 15 below.

Our strategy for obtaining these explicit upper bounds for $|L(1, \chi)|$ is as follows.

First, we start with the integral representations of $L$-functions $L(s, \chi)$ (used to prove their functional equations) and use inverse Mellin transforms to obtain bounds on $|L(1, \chi)|$ as integrals on the vertical line $\Re(s)=c>1$ of the complex plane of complex-valued functions (see Proposition 5).

Second, we move these vertical lines of integration leftwards to $\Re(s)=$ $-1 / 2$. In the process we pick up residues (see Lemma 7 ), which yields the main part of our upper bounds.

Finally, to complete the proof of Theorem 1, we give an explicit bound on the modulus of these integrals on the line $\Re(s)=-1 / 2$ (see Lemma 8). 


\subsection{Integral representations for bounds on $L(1, \chi)$}

Lemma 3 (see [Dav, Chapter 9] or [Kob, Chapter II, Section 4, Problems 4 and 6]). Let $\chi$ be a primitive Dirichlet character modulo $f>1$. Set

$$
\begin{aligned}
A=A(\chi) & := \begin{cases}0 & \text { if } \chi(-1)=+1, \text { i.e. if } \chi \text { is even, } \\
1 & \text { if } \chi(-1)=-1, \text { i.e. if } \chi \text { is odd },\end{cases} \\
\Lambda(s, \chi) & :=\left(\frac{f}{\pi}\right)^{(s+A) / 2} \Gamma\left(\frac{s+A}{2}\right) L(s, \chi), \\
\theta(t, \chi) & :=\sum_{n \geq 1} n^{A} \chi(n) e^{-\pi n^{2} t / f} \quad(t>0), \\
W(\chi) & :=\frac{\tau(\chi)}{i^{A} \sqrt{f}}=\frac{1}{i^{A} \sqrt{f}} \sum_{n=1}^{f} \chi(n) e^{2 \pi n i / f}
\end{aligned}
$$

Then $|W(\chi)|=1$ and

$$
\theta(1 / t, \chi)=W(\chi) t^{A} \sqrt{t} \theta(t, \bar{\chi})
$$

and for $\Re(s)>1$ we have

$$
\Lambda(s, \chi)=\int_{1}^{\infty}\left\{\theta(t, \chi) t^{(s+A) / 2}+W(\chi) \theta(t, \bar{\chi}) t^{(1-s+A) / 2}\right\} \frac{d t}{t} .
$$

This integral converges absolutely for all complex $s$ and provides us with an entire continuation of $\Lambda(s, \chi)$ over the whole complex plane, and $\Lambda(s, \chi)$ satisfies the functional equation

$$
W(\chi) \Lambda(1-s, \bar{\chi})=\Lambda(s, \chi)
$$

Lemma 4.

(i) (see [Rad, Section 21]) In any strip $a \leq \sigma:=\Re(s) \leq b$ and $|t| \geq 1$ we have

$$
|\Gamma(\sigma+i t)|=O\left(e^{-\pi|t| / 2}|t|^{\sigma-1 / 2}\right) .
$$

(ii) For $\alpha>0, c>\beta$ and $c>0$,

$$
\begin{aligned}
\int_{1}^{\infty} e^{-\alpha t} t^{\beta} \frac{d t}{t} & =\frac{1}{2 i \pi} \int_{c-i \infty}^{c+i \infty} \frac{\Gamma(s) \alpha^{-s}}{s-\beta} d s, \\
\int_{1}^{\infty} e^{-\alpha t} t^{\beta}(\log t) \frac{d t}{t} & =\frac{1}{2 i \pi} \int_{c-i \infty}^{c+i \infty} \frac{\Gamma(s) \alpha^{-s}}{(s-\beta)^{2}} d s .
\end{aligned}
$$

Proof. Since $\Gamma(s)=\int_{0}^{\infty} e^{-t} t^{s-1} d t$ is the Mellin transform of $s \mapsto e^{s}$, we see that

$$
e^{-x}=\frac{1}{2 i \pi} \int_{c-i \infty}^{c+i \infty} \Gamma(s) x^{-s} d s, \quad c>0,
$$


is the inverse Mellin transform of $\Gamma(s)$ (a direct proof can also be obtained by shifting the vertical line of integration $\Re(s)=c>1$ leftwards to $\Re(s)=-\infty$ (use (6) to justify this displacement) and by noticing that $\left.\operatorname{Res}_{s=-n}\left(\Gamma(s) x^{-s}\right)=(-x)^{n} / n !\right)$. This integral is absolutely convergent, by (6), and Fubini's theorem yields

$$
\begin{aligned}
\int_{1}^{\infty} e^{-\alpha t} t^{\beta-1} d t & =\int_{1}^{\infty}\left(\frac{1}{2 i \pi} \int_{c-i \infty}^{c+i \infty} \Gamma(s)(\alpha t)^{-s} d s\right) t^{\beta-1} d t \\
& =\frac{1}{2 i \pi} \int_{c-i \infty}^{c+i \infty} \Gamma(s) \alpha^{-s}\left(\int_{1}^{\infty} t^{\beta-1-s} d t\right) d s \\
& =\frac{1}{2 i \pi} \int_{c-i \infty}^{c+i \infty} \Gamma(s) \alpha^{-s} \frac{d s}{s-\beta}
\end{aligned}
$$

for $c=\Re(s)>\beta$. The proof of the second assertion is similar.

\section{Proposition 5.}

(i) Set

$$
I_{A}(f):=\frac{1}{2 i \pi} \int_{c-i \infty}^{c+i \infty} h_{A}(s) d s \quad(c>1)
$$

with

$$
h_{A}(s):=(f / \pi)^{s} \zeta(2 s-A) \Gamma(s)\left(\frac{1}{s-(1+A) / 2}+\frac{1}{s-A / 2}\right),
$$

and

$$
\widetilde{I}_{A}(f):=\frac{1}{2 i \pi} \int_{c-i \infty}^{c+i \infty} \widetilde{h}_{A}(s) d s \quad(c>1)
$$

with

$$
\widetilde{h}_{A}(s):=(f / \pi)^{s} \zeta(2 s-A) \Gamma(s)\left(\frac{1}{(s-(1+A) / 2)^{2}}-\frac{1}{(s-A / 2)^{2}}\right) .
$$

Then, for given $\sigma_{1}<0$ and $\sigma_{2}>1$, there exists $C>0$ such that in the range $\sigma_{1} \leq \sigma \leq \sigma_{2}$ and $|t| \geq 1$ we have $\left|h_{A}(\sigma+i t)\right|=$ $O\left(|t|^{C} e^{-\pi|t| / 2}\right)$ and $\left|\widetilde{h}_{A}(\sigma+i t)\right|=O\left(|t|^{C} e^{-\pi|t| / 2}\right)$.

(ii) Let $\chi$ be a primitive Dirichlet character modulo $f>1$. Then

$$
|\Lambda(1, \chi)| \leq I_{A}(f) .
$$

Moreover, if $\chi$ is quadratic, then $0<\beta<1$ and $L(\beta, \chi)=0$ imply

$$
|\Lambda(1, \chi)| \leq \frac{1-\beta}{2} \widetilde{I}_{A}(f) .
$$


Proof. The first assertion follows from (6) and (5) (see [Lou00, proof of Lemma 12]). To prove (9), we use (4) for $s=1$, and Lemma 4 . To prove (10), we notice that $L(\beta, \chi)=0$ implies $\Lambda(\beta, \chi)=0$ and

$$
|\Lambda(1, \chi)|=|\Lambda(1, \chi)-\Lambda(\beta, \chi)| \leq(1-\beta) \max _{\beta \leq s \leq 1}\left|\Lambda^{\prime}(s, \chi)\right| .
$$

Now, we first take the derivative of the integral representation of $\Lambda(s, \chi)$ given in (4). Second, we notice that if $\chi$ is quadratic then $\bar{\chi}=\chi$ and $W(\chi)=+1$. Third, due to the functional equation (5), we may assume that $1 / 2 \leq \beta \leq 1$. Fourth, for $t \geq 1$ real the function

$$
s \mapsto t^{(s+A) / 2-1}-t^{(1-s+A) / 2-1}
$$

increases with $s$, and is nonnegative in the range $s \geq 1 / 2$. Therefore, for $1 / 2 \leq s \leq 1$ we have

$$
2\left|\Lambda^{\prime}(s, \chi)\right| \leq \int_{1}^{\infty}\left(\sum_{n \geq 1} n^{A} e^{-\pi n^{2} t / f}\right)(\log t)\left(t^{(1+A) / 2}-t^{A / 2}\right) \frac{d t}{t},
$$

and we use Lemma 4 to complete the proof.

Now, set

$$
J_{A}(f)=\frac{1}{2 i \pi} \int_{-1 / 2-i \infty}^{-1 / 2+i \infty} h_{A}(s) d s, \quad \widetilde{J}_{A}(f)=\frac{1}{2 i \pi} \int_{-1 / 2-i \infty}^{-1 / 2+i \infty} \widetilde{h}_{A}(s) d s .
$$

According to Proposition 5(i), we are allowed to move the vertical lines of integration $\Re(s)=c>1$ in (7) and (8) leftwards to $\Re(s)=-1 / 2$. We pick up residues at $s=(1+A) / 2, s=A / 2$ and $s=0$ and obtain:

Lemma 6. Let $\chi$ be a primitive Dirichlet character modulo $f>1$. If $\chi$ is even, then

$$
\begin{aligned}
\sqrt{f}|L(1, \chi)| & =|\Lambda(1, \chi)| \\
& \leq I_{0}(f)=\operatorname{Res}_{s=1 / 2}\left(h_{0}(s)\right)+\operatorname{Res}_{s=0}\left(h_{0}(s)\right)+J_{0}(f) .
\end{aligned}
$$

If $\chi$ is odd, then

$$
\begin{aligned}
\frac{f}{\pi}|L(1, \chi)| & =|\Lambda(1, \chi)| \leq I_{1}(f) \\
& =\operatorname{Res}_{s=1}\left(h_{0}(s)\right)+\operatorname{Res}_{s=1 / 2}\left(h_{0}(s)\right)+\operatorname{Res}_{s=0}\left(h_{0}(s)\right)+J_{1}(f) .
\end{aligned}
$$

If $\chi$ is even and $L(\beta, \chi)=0$ for some $\beta \in(0,1)$, then

$\sqrt{f}|L(1, \chi)| \leq \frac{1-\beta}{2} \widetilde{I}_{0}(f)=\frac{1-\beta}{2}\left(\operatorname{Res}_{s=1 / 2}\left(\widetilde{h}_{0}(s)\right)+\operatorname{Res}_{s=0}\left(\widetilde{h}_{0}(s)\right)+\widetilde{J}_{0}(f)\right)$. 
If $\chi$ is odd and $L(\beta, \chi)=0$ for some $\beta \in(0,1)$, then

$$
\begin{aligned}
\frac{f}{\pi}|L(1, \chi)| & \leq \frac{1-\beta}{2} \widetilde{I}_{1}(f) \\
& =\frac{1-\beta}{2}\left(\operatorname{Res}_{s=1}\left(\widetilde{h}_{0}(s)\right)+\operatorname{Res}_{s=1 / 2}\left(\widetilde{h}_{0}(s)\right)+\operatorname{Res}_{s=0}\left(\widetilde{h}_{0}(s)\right)+\widetilde{J}_{1}(f)\right) .
\end{aligned}
$$

Now, the remainder of the proof is easy: we compute these residues (see Lemma 7) and find explicit bounds for $\left|J_{A}(f)\right|$ and $\left|\widetilde{J}_{A}(f)\right|$ (see Lemma 8).

2.2. The residues of $h_{A}(s)$ and $\widetilde{h}_{A}(s)$, and bounds for $J_{A}(f)$ and $\widetilde{J}_{A}(f)$. Let $\kappa_{0}$ and $\kappa_{1}$ be as in $(1)$ and set

$$
\left\{\begin{array}{l}
\gamma(1)=\lim _{m \rightarrow \infty}\left(\sum_{k=1}^{m} \frac{\log k}{k}-\frac{1}{2} \log ^{2} m\right)=-0.072815 \ldots \\
\kappa_{0}^{\prime}=\log (4 \pi)-\gamma=1.953808 \ldots \\
\kappa_{0}^{\prime \prime}=\pi^{2} / 2-8-4 \gamma^{2}-8 \gamma(1)=-3.815382 \ldots \\
\kappa_{1}^{\prime}=\log \pi-\gamma=0.567514 \ldots \\
\kappa_{1}^{\prime \prime}=\pi^{2} / 6-8-4 \gamma^{2}-8 \gamma(1)=-7.105250 \ldots
\end{array}\right.
$$

Lemma 7. Let the notation be as in Proposition 5. We have

$$
\begin{aligned}
& \left\{\begin{array}{l}
\operatorname{Res}_{s=1 / 2}\left(h_{0}(s)\right)=\frac{1}{2} \sqrt{f}\left(\log f+\kappa_{0}\right), \\
\operatorname{Res}_{s=0}\left(h_{0}(s)\right)=-\frac{1}{2}\left(\log f-\kappa_{0}\right) .
\end{array}\right. \\
& \left\{\begin{array}{l}
\operatorname{Res}_{s=1}\left(h_{1}(s)\right)=\frac{f}{2 \pi}\left(\log f+\kappa_{1}\right), \\
\operatorname{Res}_{s=1 / 2}\left(h_{1}(s)\right)=-\frac{1}{2} \sqrt{f}, \\
\operatorname{Res}_{s=0}\left(h_{1}(s)\right)=\frac{1}{4} \cdot
\end{array}\right. \\
& \left\{\begin{array}{l}
\operatorname{Res}_{s=1 / 2}\left(\widetilde{h}_{0}(s)\right)=\frac{1}{4} \sqrt{f}\left(\left(\log f-\kappa_{0}^{\prime}\right)^{2}+\kappa_{0}^{\prime \prime}\right), \\
\operatorname{Res}_{s=0}\left(\widetilde{h}_{0}(s)\right)=\frac{1}{4}\left(\left(\log f+\kappa_{0}^{\prime}\right)^{2}+\kappa_{0}^{\prime \prime}\right) .
\end{array}\right. \\
& \left\{\begin{array}{l}
\operatorname{Res}_{s=1}\left(\widetilde{h}_{1}(s)\right)=\frac{f}{4 \pi}\left(\left(\log f-\kappa_{1}^{\prime}\right)^{2}+\kappa_{1}^{\prime \prime}\right), \\
\operatorname{Res}_{s=1 / 2}\left(\widetilde{h}_{1}(s)\right)=\frac{1}{2} \sqrt{f}\left(\log f+\kappa_{1}^{\prime}\right), \\
\operatorname{Res}_{s=0}\left(\widetilde{h}_{1}(s)\right)=\frac{1}{4} .
\end{array}\right.
\end{aligned}
$$


Proof. Use

$$
\begin{aligned}
\zeta(1+\varepsilon) & =\frac{1}{\varepsilon}+\gamma-\gamma(1) \varepsilon+O\left(\varepsilon^{2}\right), \\
\zeta(\varepsilon) & =-\frac{1}{2}-\frac{\log (2 \pi)}{2} \varepsilon-\left(\frac{\log ^{2}(2 \pi)}{4}+\frac{\pi^{2}}{48}-\frac{\gamma(1)}{2}-\frac{\gamma^{2}}{4}\right) \varepsilon^{2}+O\left(\varepsilon^{3}\right), \\
\frac{\Gamma((1+\varepsilon) / 2)}{\Gamma(1 / 2)} & =1-\frac{\gamma+\log 4}{2} \varepsilon+\left(\frac{\pi^{2}}{16}+\frac{(\gamma+\log 4)^{2}}{8}\right) \varepsilon^{2}+O\left(\varepsilon^{3}\right), \\
\Gamma(1+\varepsilon) & =1-\gamma \varepsilon+\frac{\gamma^{2}+\pi^{2} / 6}{2} \varepsilon^{2}+O\left(\varepsilon^{3}\right),
\end{aligned}
$$

and $\Gamma(1 / 2)=\sqrt{\pi}, \zeta(0)=-1 / 2$ and $\zeta(-1)=-1 / 12$ (use (13) below).

LEMMA 8. We have

$\left|J_{0}(f)\right| \leq \sqrt{\frac{4 \pi}{9 f}} K_{0} \quad$ with $K_{0}:=\int_{0}^{\infty} \sqrt{\frac{t}{\left(1+4 t^{2}\right) \sinh (\pi t)}} d t=0.414872 \ldots$,

$\left|J_{1}(f)\right| \leq \frac{2 \zeta(3)}{\sqrt{\pi^{5} f}} K_{1}$ with $K_{1}:=\int_{0}^{\infty} \sqrt{\frac{t \sinh (\pi t)}{\cosh ^{2}(\pi t)}} d t=0.571232 \ldots$,

$\left|\widetilde{J}_{0}(f)\right| \leq \sqrt{\frac{16 \pi}{9 f}} \widetilde{K}_{0}$ with $\widetilde{K}_{0}:=\int_{0}^{\infty} \sqrt{\frac{t}{\left(1+4 t^{2}\right)^{2} \sinh (\pi t)}} d t=0.296996 \ldots$,

$\left|\widetilde{J}_{1}(f)\right| \leq \frac{2 \zeta(3)}{\sqrt{\pi^{5} f}} \widetilde{K}_{1}$ with $\widetilde{K}_{1}:=\int_{0}^{\infty} \sqrt{\frac{t \sinh (\pi t)}{\left(1+t^{2}\right) \cosh ^{2}(\pi t)}} d t=0.416696 \ldots$

Proof. Using $\overline{\Gamma(s)}=\Gamma(\bar{s})$ and $\Gamma(s) \Gamma(1-s)=\pi / \sin (\pi s)$ we obtain $|\Gamma(1 / 2+i t)|=\sqrt{\pi / \cosh (\pi t)}$. Using

$$
\frac{1}{\Gamma(s)}=s e^{\gamma s} \prod_{n \geq 1}\left(1+\frac{s}{n}\right) e^{-s / n} \quad \text { and } \quad \sin (\pi s)=\pi s \prod_{n \geq 1}\left(1-\frac{s^{2}}{n^{2}}\right)
$$

we obtain $|\Gamma(i t)|=\sqrt{\pi / t \sinh (\pi t)}$ and $|\Gamma(1+i t)|=|i t \Gamma(i t)|=\sqrt{\pi t / \sinh (\pi t)}$. Hence, using the well known functional equation

$$
\zeta(1-s)=2(2 \pi)^{-s} \cos (\pi s / 2) \Gamma(s) \zeta(s),
$$

we deduce that for $s=-1 / 2+i t$ we have

$$
\left|(f / \pi)^{s} \zeta(2 s) \Gamma(s)\right|=\frac{1}{\sqrt{\pi f}} \sqrt{\frac{t}{\sinh (\pi t)}}|\zeta(2-2 i t)|
$$

and

$$
\left|(f / \pi)^{s} \zeta(2 s-1) \Gamma(s)\right|=\frac{|1-i t|}{\sqrt{\pi^{3} f}} \sqrt{\frac{t \sinh (\pi t)}{\cosh ^{2}(\pi t)}}|\zeta(3-2 i t)|,
$$

from which the desired results follow. 
By moving the vertical lines of integration $\Re(s)=c>1$ in (7) and (8) leftwards to $\Re(s)=-1 / 2$ and by using Lemmas 7 and 8 , we obtain the following result which completes the proof of Theorem 1 (use Lemma 6):

Lemma 9. Let the notation be as in Lemma 8. Let $\theta$ stand for a real number with $|\theta| \leq 1$. Then

$$
\begin{aligned}
I_{0}(f)= & \frac{1}{2} \sqrt{f}\left(\log f+\kappa_{0}\right)-\frac{1}{2}\left(\log f-\kappa_{0}\right)+\theta \sqrt{\frac{4 \pi}{9 f}} K_{0} \\
\leq & \frac{1}{2} \sqrt{f}\left(\log f+\kappa_{0}\right) \quad \text { for } f \geq 5, \\
I_{1}(f)= & \frac{f}{2 \pi}\left(\log f+\kappa_{1}\right)-\frac{1}{2} \sqrt{f}+\frac{1}{4}+\theta \frac{2 \zeta(3)}{\sqrt{\pi^{5} f}} K_{1} \\
\leq & \frac{f}{2 \pi}\left(\log f+\kappa_{1}\right) \quad \text { for } f \geq 3, \\
\widetilde{I}_{0}(f)= & \frac{1}{4} \sqrt{f}\left(\left(\log f-\kappa_{0}^{\prime}\right)^{2}+\kappa_{0}^{\prime \prime}\right)+\frac{1}{4}\left(\left(\log f+\kappa_{0}^{\prime}\right)^{2}+\kappa_{0}^{\prime \prime}\right) \\
& +\theta \sqrt{\frac{16 \pi}{9 f}} \widetilde{K}_{0} \\
\leq & \frac{1}{4} \sqrt{f} \log ^{2} f \quad \text { for } f \geq 5, \\
\widetilde{I}_{1}(f)= & \frac{f}{4 \pi}\left(\left(\log ^{\prime} f-\kappa_{1}^{\prime}\right)^{2}+\kappa_{1}^{\prime \prime}\right)+\frac{1}{2} \sqrt{f}\left(\log f+\kappa_{1}^{\prime}\right) \\
& +\frac{1}{4}+\theta \frac{2 \zeta(3)}{\sqrt{\pi^{5} f}} \widetilde{K}_{1} \\
\leq & \frac{f}{4 \pi} \log ^{2} f \quad \text { for } f \geq 3 .
\end{aligned}
$$

REMARK 10. To prove Corollary 11 below we need the following more complicated bound: if $\chi$ is a primitive, even Dirichlet character modulo $f>1$, then $0<\beta<1$ and $L(\beta, \chi)=0$ imply

$$
0<L(1, \chi) \leq \frac{1-\beta}{8}(\log f)(2 \log ((\sqrt{f-4}+\sqrt{f}) / 2))
$$

(for the right hand side of $(16)$ is $\leq \frac{1}{4} \sqrt{f}(\log f)(2 \log ((\sqrt{f-4}+\sqrt{f}) / 2))$ for $f \geq 5$ ).

\subsection{Corollaries to Theorem 1}

Corollary 11. Let $L$ be a quadratic number field. Let $d_{L}$ denote the absolute value of the discriminant of $L$. Then

$\zeta_{L}(s)<0 \quad$ for $s \in \begin{cases}(0,1) \cap\left[1-\left(8 \pi / \sqrt{d_{L}} \log ^{2} d_{L}\right), 1\right) & \text { if } L \text { is imaginary }, \\ (0,1) \cap\left[1-\left(8 / \sqrt{d_{L}} \log d_{L}\right), 1\right) & \text { if } L \text { is real. }\end{cases}$ 
Proof. Let $\chi_{L}, h_{L}, w_{L}$ denote the quadratic primitive Dirichlet character modulo $d_{L}$ associated with $L$, the class number of $L$ and the number of complex roots of unity in $L$, respectively. First, assume that $L$ is imaginary. Then $0<\beta<1$ and $\zeta_{L}(\beta)=0$ imply

$$
1 \leq h_{L}=\frac{w_{L} \sqrt{d_{L}}}{2 \pi} L\left(1, \chi_{L}\right) \leq(1-\beta) \frac{w_{L} \sqrt{d_{L}}}{16 \pi} \log ^{2} d_{L},
$$

by (3), hence $\beta \leq 1-16 \pi / w_{L} \sqrt{d_{L}} \log ^{2} d_{L}$. Since this latter bound is $\leq 0$ for $d_{L}=3$ (in which case $w_{L}=6$ ) and $d_{L}=4$ (in which case $w_{L}=4$ ), and since $w_{L}=2$ otherwise, the desired result follows. Now, assume that $L$ is real and let $\varepsilon_{L}=\left(x_{L}+y_{L} \sqrt{d_{L}}\right) / 2>1$ denote the fundamental unit of $L$ (with $x_{L} \geq 1$ and $y_{L} \geq 1$ some positive integers satisfying $x_{L}^{2}-d_{L} y_{L}^{2}= \pm 4$ ). Then $\varepsilon_{L}=\left(\sqrt{d_{L} y_{L}^{2} \pm 4}+y_{L} \sqrt{d_{L}}\right) / 2 \geq\left(\sqrt{d_{L}-4}+\sqrt{d_{L}}\right) / 2$, and $0<\beta<1$ and $\zeta_{L}(\beta)=0$ imply

$$
\begin{aligned}
1 & \leq h_{L}=\frac{\sqrt{d_{L}}}{2 \log \varepsilon_{L}} L\left(1, \chi_{L}\right) \\
& \leq \frac{\sqrt{d_{L}}}{2 \log \left(\left(\sqrt{d_{L}-4}+\sqrt{d_{L}}\right) / 2\right)} L\left(1, \chi_{L}\right) \leq \frac{1-\beta}{8} \sqrt{d_{L}} \log d_{L},
\end{aligned}
$$

by (18), hence $\beta \leq 1-8 / \sqrt{d_{L}} \log d_{L}$.

REMARK 12. Better results with more complicated proofs can be found in [Bes, Theorem 17] and [Pin, (1.12)].

Corollary 13. Let $K$ be a real abelian number field of degree $n>1$. Let $X_{K}$ denote the group of order $n$ of primitive Dirichlet characters associated with $K$. Then

$$
\operatorname{Res}_{s=1}\left(\zeta_{K}(s)\right) \leq \prod_{1 \neq \chi \in X_{K}} \frac{1}{2}\left(\log f_{\chi}+\kappa_{0}\right),
$$

and if $\zeta_{L}(\beta)=0$ for some $\beta \in(0,1)$ and some real quadratic subfield $L$ of $K$ then

$$
\operatorname{Res}_{s=1}\left(\zeta_{K}(s)\right) \leq \frac{1-\beta}{4}\left(\log f_{L}\right) \prod_{1 \neq \chi \in X_{K}} \frac{1}{2}\left(\log f_{\chi}+\kappa_{0}\right) .
$$

Proof. $\operatorname{Res}_{s=1}\left(\zeta_{K}(s)\right)=\prod_{1 \neq \chi \in X_{K}} L(1, \chi)$.

For nonabelian number fields, we have a weaker result:

TheOREm 14 (see [Lou01, Theorem 1]). Let $K$ be a number field of degree $n>1$. Let $d_{K}$ denote the absolute value of the discriminant of $K$. Then

$$
\operatorname{Res}_{s=1}\left(\zeta_{K}(s)\right) \leq\left(\frac{e \log d_{K}}{2(n-1)}\right)^{n-1},
$$


and if $\zeta_{K}(\beta)=0$ for some $\beta \in(0,1)$ then

$$
\operatorname{Res}_{s=1}\left(\zeta_{K}(s)\right) \leq(1-\beta)\left(\frac{e \log d_{K}}{2 n}\right)^{n} .
$$

\subsection{Upper bounds for $|L(1, \chi)|$ : continued}

TheOREM 15. Let $\chi$ be a primitive Dirichlet character modulo $f>1$ if $\chi$ is even, and modulo $f>820$ if $\chi$ is odd. Then $0<\beta<1$ and $L(\beta, \chi)=0$ imply

$$
|L(1, \chi)| \leq \frac{1-\beta}{8} \log ^{2} f .
$$

Proof. The proof is similar to that of Theorem 1, apart from the fact that since in this case we do not have $W(\chi)=1$, we start with the bound

$$
2\left|\Lambda^{\prime}(s, \chi)\right| \leq \int_{1}^{\infty}\left(\sum_{n \geq 1} n^{A} e^{-\pi n^{2} t / f}\right)(\log t)\left(t^{(1+A) / 2}+t^{A / 2}\right) \frac{d t}{t}
$$

(slightly weaker than (11)). We obtain $|\Lambda(1, \chi)| \leq \frac{1-\beta}{2} \widetilde{I}_{A}(f)$ where

$$
\widetilde{I}_{A}(f):=\frac{1}{2 i \pi} \int_{c-i \infty}^{c+i \infty} \widetilde{h}_{A}(s) d s
$$

with

$$
\widetilde{h}_{A}(s):=(f / \pi)^{s} \zeta(2 s-A) \Gamma(s)\left(\frac{1}{(s-(1+A) / 2)^{2}}+\frac{1}{(s-A / 2)^{2}}\right) .
$$

With $\kappa_{0}^{\prime}$ and $\kappa_{1}^{\prime}$ as in (12) and setting

$$
\left\{\begin{array}{l}
\kappa_{0}^{\prime \prime \prime}=\kappa_{0}^{\prime \prime}+16=\pi^{2} / 2+8-4 \gamma^{2}-8 \gamma(1)=12.184617 \ldots \\
\kappa_{1}^{\prime \prime \prime}=\kappa_{1}^{\prime \prime}+16=\pi^{2} / 6+8-4 \gamma^{2}-8 \gamma(1)=8.8947491 \ldots
\end{array}\right.
$$

we have

$$
\begin{aligned}
& \widetilde{I}_{0}(f)=\frac{1}{4} \sqrt{f}\left(\left(\log f-\kappa_{0}^{\prime}\right)^{2}+\kappa_{0}^{\prime \prime \prime}\right)-\frac{1}{4}\left(\left(\log f+\kappa_{0}^{\prime}\right)^{2}+\kappa_{0}^{\prime \prime \prime}\right)+\theta \sqrt{\frac{16 \pi}{9 f}} \widetilde{K}_{0}, \\
& \widetilde{I}_{1}(f)=\frac{f}{4 \pi}\left(\left(\log f-\kappa_{1}^{\prime}\right)^{2}+\kappa_{1}^{\prime \prime \prime}\right)-\frac{1}{2} \sqrt{f}\left(\log f+\kappa_{1}^{\prime}\right)-\frac{5}{12}+\theta \frac{2 \zeta(3)}{\sqrt{\pi^{5} f}} \widetilde{K}_{1}
\end{aligned}
$$

(to be compared with (16) and (17)), with $|\theta| \leq 1$ (and where $\widetilde{K}_{0}$ and $\widetilde{K}_{1}$ are as in Lemma 8). The desired result follows.

We refer the reader to [BL] and [Lou01, Theorem 18] for an application of this result. 


\section{LOWER BOUNDS FOR RELATIVE CLASS NUMBERS}

The aim of this section is to obtain, in Theorems 18, 28 and 31, explicit lower bounds for the relative class numbers $h_{K}^{-}$of CM-fields $K$. Let $K$ be a CM-field of degree $2 n \geq 2$ and let $K^{+}$denote its maximal totally real subfield (of degree $n$ ). The class number $h_{K^{+}}$of $K^{+}$divides the class number $h_{K}$ of $K$, and (see [Has] or [Was])

$$
h_{K}^{-}:=h_{K} / h_{K^{+}}=\frac{Q_{K} w_{K}}{(2 \pi)^{n}} \sqrt{\frac{d_{K}}{d_{K^{+}}}} \frac{\operatorname{Res}_{s=1}\left(\zeta_{K}(s)\right)}{\operatorname{Res}_{s=1}\left(\zeta_{K^{+}}(s)\right)} .
$$

Here $Q_{K}:=\left(E_{K}: W_{K} E_{K^{+}}\right) \in\{1,2\}$ is the Hasse unit index of $K$ (where $E_{K}$ and $E_{K^{+}}$are the unit groups of $K$ and $K^{+}$, and $W_{K}$ is the group of roots of unity in $K), w_{K}:=\# W_{K} \geq 2$ is the number of roots of unity in $K$, and $d_{K}$ and $d_{K^{+}}$are the absolute values of the discriminants of $K$ and $K_{+}$, respectively. In particular, if $K$ is an imaginary abelian number field, we obtain

$$
h_{K}^{-}=\frac{Q_{K} w_{K}}{(2 \pi)^{n}} \sqrt{\frac{d_{K}}{d_{K^{+}}}} \prod_{\chi \in X_{K}^{-}} L(1, \chi),
$$

where $X_{K}^{-}=\left\{\chi \in X_{K} ; \chi(-1)=-1\right\}$ is of cardinality $n$. Formulae (21) and (22) are useful for obtaining upper and lower bounds for relative class numbers.

THEOREM 16.

(i) Let $K$ be an imaginary abelian number field of degree $2 n$. Then

$$
h_{K}^{-} \leq \frac{Q_{K} w_{K}}{(4 \pi)^{n}} \sqrt{d_{K} / d_{K^{+}}}\left(\frac{1}{n} \log \left(d_{K} / d_{K^{+}}\right)+\kappa_{1}\right)^{n} .
$$

(ii) (see [Lou00, Corollary 3]). Let $K$ be a CM-field of degree $2 n$. Then

$$
h_{K}^{-} \leq 2 Q_{K} w_{K} \sqrt{d_{K} / d_{K^{+}}}\left(\frac{e}{4 \pi n} \log \left(d_{K} / d_{K^{+}}\right)\right)^{n} .
$$

Proof. To prove (23), use (22), Theorem 1, the fact that the geometric mean is less than or equal to the arithmetic mean, that $\# X_{K}^{-}=n$ and that $d_{K} / d_{K^{+}}=\prod_{\chi \in X_{K}^{-}} f_{\chi}$

Lemma 17 (see [Lou03, Theorem 1], cf. [Lou94, Proposition A]).

(i) Let $N$ be a totally imaginary number field of degree $2 n \geq 4$ and root discriminant $\varrho_{N}:=d_{N}^{1 / 2 n} \geq 50$.

(a) If $\zeta_{N}(\beta) \leq 0$ for some $\beta \in\left[1-2 / \log d_{N}, 1\right)$, then

$$
\operatorname{Res}_{s=1}\left(\zeta_{N}(s)\right) \geq \frac{1}{2}(1-\beta) d_{N}^{(\beta-1) / 2} .
$$


(b) If $\zeta_{N}(\beta) \leq 0$ for some $\beta \in\left[1-2 / \log d_{N}, 1-1 / \log d_{N}\right]$, then

$$
\operatorname{Res}_{s=1}\left(\zeta_{N}(s)\right) \geq(1-\beta) d_{N}^{(\beta-1) / 2} .
$$

(ii) Let $K$ be a totally imaginary number field of degree $2 n>2$ and root discriminant $\varrho_{K} \geq 2683$. Assume that $\zeta_{K}(\beta) \leq 0$ for some $\beta \in\left[1-2 / \log d_{K}, 1\right)$. Then

$$
\kappa_{K} \geq(1-\beta) d_{K}^{(\beta-1) / 2}
$$

\subsection{The abelian case}

TheOREM 18. Let $N$ be an imaginary abelian number field of degree $2 n \geq 4$, conductor $f_{N}$ and root discriminant $\varrho_{N} \geq 50$.

(i) If $N$ contains an imaginary quadratic subfield $L$ for which $\zeta_{L}(\beta)=0$ for some $\beta \in\left[1-2 / \log d_{N}, 1\right)$, then

which implies

$$
h_{N}^{-} / h_{L} \geq \frac{2 Q_{N} w_{N} \sqrt{d_{N} / d_{N^{+}} d_{L}}}{e\left(\log ^{2} d_{L}\right) \prod_{1 \neq \chi \in X_{N}^{+}} \pi\left(\log f_{\chi}+\kappa_{0}\right)},
$$

(ii) Otherwise,

which implies

$$
h_{N}^{-} \geq \frac{4 \sqrt{d_{N} / d_{N^{+}}}}{e \pi^{n}\left(\log f_{N}+\kappa_{0}\right)^{n}} \cdot \frac{\pi h_{L}}{\sqrt{d_{L}} \log d_{L}} .
$$

$$
h_{N}^{-} \geq \frac{Q_{N} w_{N} \sqrt{d_{N} / d_{N^{+}}}}{e \pi\left(\log d_{N}\right) \prod_{1 \neq \chi \in X_{N}^{+}} \pi\left(\log f_{\chi}+\kappa_{0}\right)},
$$

Proof. (i) Since $\zeta_{L}(\beta)=0$ implies $\zeta_{N}(\beta)=0$, we have

$$
\operatorname{Res}_{s=1}\left(\zeta_{N}(s)\right) \geq \frac{1}{2}(1-\beta) d_{N}^{(\beta-1) / 2} \geq \frac{1-\beta}{2 e},
$$

by (25), and

$$
h_{N}^{-} \geq \frac{2 \sqrt{d_{N} / d_{N^{+}}}}{(2 n-1) e \pi^{n}\left(\log f_{N}+\kappa_{0}\right)^{n}} .
$$


by Corollary 13. Hence, using (21) for $N$ and $L$ and noticing $Q_{L}=\left(W_{L}: W_{L}\right)$ $=1, w_{L}=2$ (for the Dedekind zeta functions $\zeta_{L}(s)$ of $L=\mathbb{Q}(\sqrt{-1})$ and $L=\mathbb{Q}(\sqrt{-3})$ have no zero in $(0,1))$ and $\operatorname{Res}_{s=1}\left(\zeta_{L^{+}}(s)\right)=\operatorname{Res}_{s=1}(\zeta(s))=1$, we obtain (28).

(ii) To begin with, we notice that

$$
\begin{aligned}
& \zeta_{N}(s) \\
& = \begin{cases}\zeta(s)\left(\prod_{\substack{1 \neq \chi \in X_{N} \\
\chi^{2}=1}} L(s, \chi)\right)\left(\prod_{\substack{\chi \in X_{N} \\
\chi^{2} \neq 1}} L(s, \chi)\right) & (s \text { complex }), \\
\zeta(s)\left(\prod_{\substack{L \subseteq N \\
L \text { quadratic }}} \zeta_{L}(s) / \zeta(s)\right)\left(\prod_{\substack{\{\chi, \bar{\chi}\} \subseteq X_{N} \\
\chi^{2} \neq 1}}|L(s, \chi)|^{2}\right) & (s \text { real })\end{cases}
\end{aligned}
$$

(for the nonquadratic characters $\chi \in X_{N}$ come in conjugate pairs $\{\chi, \bar{\chi}\}$ with $\bar{\chi} \neq \chi$, and $L(s, \chi) L(s, \bar{\chi})=|L(s, \chi)|^{2} \geq 0$ for $s$ real). Recall that if $\chi \neq 1$ is a nontrivial primitive Dirichlet character, then $L(s, \chi)$ is entire, and that using

$$
\sum_{n \geq 1} \frac{(-1)^{n-1}}{n^{s}}=\left(1-2^{1-s}\right) \zeta(s) \quad(\Re(s)>0),
$$

we obtain $\zeta(s)<0$ for $0<s<1$.

(a) Assume that $N$ contains a real quadratic subfield $L$ for which $\zeta_{L}(\beta)$ $=0$ for some $\beta \in\left[1-2 / \log d_{N}, 1\right)$. Then $\zeta_{N^{+}}(\beta)=\zeta_{N}(\beta)=0$,

by (25), and

$$
\operatorname{Res}_{s=1}\left(\zeta_{N}(s)\right) \geq \frac{1}{2}(1-\beta) d_{N}^{(\beta-1) / 2} \geq \frac{1-\beta}{2 e}
$$

$$
\operatorname{Res}_{s=1}\left(\zeta_{N^{+}}(s)\right) \leq \frac{1-\beta}{4}\left(\log d_{N}\right) \prod_{1 \neq \chi \in X_{N}^{+}} \frac{1}{2}\left(\log f_{\chi}+\kappa_{0}\right)
$$

by Corollary 13 (for $d_{N} \geq d_{L}^{n}=f_{L}^{n} \geq f_{L}$ for any quadratic subfield $L$ of $N$ ). Hence, using (21), we conclude that (30) is valid.

(b) It remains to consider the case that the Dedekind zeta functions $\zeta_{L}(s)$ of all the quadratic subfields $L$ of $N$ have no real zero in $\left[1-2 / \log d_{N}, 1\right)$. Then $\zeta_{N}\left(1-2 / \log d_{N}\right) \leq 0$ by $(32)$. Hence,

$$
\operatorname{Res}_{s=1}\left(\zeta_{N}(s)\right) \geq \frac{2}{e \log d_{N}}
$$

by (26). Moreover,

$$
\operatorname{Res}_{s=1}\left(\zeta_{N^{+}}(s)\right) \leq \prod_{1 \neq \chi \in X_{N}^{+}} \frac{1}{2}\left(\log f_{\chi}+\kappa_{0}\right)
$$

by Corollary 13. Hence, using (21), we deduce that (30) is here also valid. 
REMARK 19. According to the last part of this proof, we also have the following result (see Corollary 23 below for an application): if $N$ is an imaginary abelian number field and if the Dedekind zeta functions $\zeta_{L}(s)$ of all the quadratic subfields $L$ of $N$ have no real zero in $\left[1-2 / \log d_{N}, 1\right)$, then

$$
h_{N}^{-} \geq \frac{2 Q_{N} w_{N} \sqrt{d_{N} / d_{N^{+}}}}{e(2 \pi)^{n} \operatorname{Res}_{s=1}\left(\zeta_{N^{+}}(s)\right)} .
$$

Corollary $20\left({ }^{1}\right)$. Let $N$ be an imaginary cyclic number field of 2-power degree $2 n=2^{m} \geq 4$ and root discriminant $\varrho_{N} \geq 50$. Then $d_{N} / d_{N^{+}}=f_{N}^{n}$, $d_{N} \leq f_{N}^{2 n-1}$ and

$$
h_{N}^{-} \geq \frac{2}{e(2 n-1)}\left(\frac{f_{N}}{\pi^{2}\left(\log f_{N}+\kappa_{0}\right)^{2}}\right)^{n / 2},
$$

which implies $h_{N}^{-}>1$ for $f_{N} \geq 2500\left(^{2}\right)$.

Proof. Since $N$ contains no imaginary quadratic subfield, (31) is valid.

Corollary 21. Let $N$ be an imaginary cyclic field of degree $2 p(p \geq 3$ an odd prime) and root discriminant $\varrho_{N} \geq 50$. Let $L$ denote its imaginary quadratic subfield. Then $d_{N} / d_{N^{+}}=f_{L} f_{N}^{p-1}, d_{N} \leq f_{N}^{2 p-1}, h_{L}$ divides $h_{N}^{-}$(see [LOO, Theorem 5] and [Lem, Corollary 1]) and

$$
h_{N}^{-} / h_{L} \geq \varepsilon_{N}\left(\frac{\sqrt{f_{N}}}{\pi\left(\log f_{N}+\kappa_{0}\right)}\right)^{p-1},
$$

where

$$
\varepsilon_{N}:=\min \left(\frac{4}{e \log ^{2} f_{L}}, \frac{2 \sqrt{f_{L}}}{e \pi h_{L} \log d_{N}}\right) .
$$

Hence, if $h_{N}^{-}=1\left(^{3}\right)$ or if $h_{N}$ is equal to its genus class number $\left({ }^{4}\right)$, then $h_{N}^{-} / h_{L}=1$ and $f_{N} \leq 240000\left({ }^{5}\right)$.

$\left({ }^{1}\right)$ See [Lou97, Theorem 5], [Lou99] and [CK98] for applications.

$\left(^{2}\right)$ K. Uchida obtained in [Uch72, Proposition 6] the worse bound $h_{N}^{-}>1$ for $2 n=4$ and $f_{N} \geq 50000$. Whereas the lower bound (33) also implies $h_{N}^{-}>2$ for $f_{N} \geq 6300$, K. Hardy, R. Hudson, D. Richman and K. S. Williams obtained in [HHRW] the worse bound $h_{N}^{-}>2$ for $2 n=4$ and $f_{N} \geq 416000$.

$\left({ }^{3}\right)$ See [Yam] and [CK00a] for the solutions to these class number and relative class number one problems.

$\left({ }^{4}\right)$ See [Lou98a] and [CK00a] and [CK00b] for the solution to this class number problem.

$\left({ }^{5}\right)$ K. Uchida obtained in [Uch72, Proposition 7] the worse bound $h_{N}^{-}>1$ for $2 n=6$ and $f_{N} \geq 8150000$, and S. Louboutin obtained in [Lou98a, Theorem 5] the worse bound $h_{N}^{-} / h_{L} \geq 1$ for $f_{N} \geq 1300000$. 
Proof. To obtain (34), use (28), and (30) and the class number formula for $L$. Now, if $h_{N}^{-}=1$ then $h_{L}=1, \sqrt{f_{L}} / h_{L} \geq \sqrt{3}$ and

$$
1=h_{N}^{-} / h_{L} \geq \varepsilon_{N}^{\prime}\left(\frac{\sqrt{f_{N}}}{\pi\left(\log f_{N}+\kappa_{0}\right)}\right)^{p-1}
$$

where

$$
\varepsilon_{N}^{\prime}:=\min \left(\frac{4}{e \log ^{2} f_{N}}, \frac{2 \sqrt{3}}{(2 p-1) e \pi \log f_{N}}\right)
$$

(for $f_{L} \leq f_{N}$ and $d_{N} \leq f_{N}^{2 p-1}$ ), which implies $f_{N} \leq 240000$. In the same way, if $h_{N}$ is equal to its genus class number, then $h_{N}^{-} / h_{L}=1$ and $h_{L}=2^{t-1}$, where $t$ denotes the number of distinct prime divisors of $f_{L}$ (see [Lou98a, Proposition 3]). Hence, $\sqrt{f_{L}} / h_{L}=2 \sqrt{f_{L} / 4^{t}} \geq 2 \sqrt{3 / 4}=\sqrt{3}$ and here again (35) is valid.

REMARK 22. According to [Lou92], there exists some explicit constant $c>0$ such that

$$
|L(1, \chi)| \geq \frac{1}{c \log f_{\chi}}
$$

for all nonquadratic primitive Dirichlet characters $\chi$, where $f_{\chi}>1$ denotes its conductor. In particular, if $N$ is an imaginary cyclic field of degree $2 p$ ( $p \geq 3$ an odd prime) and conductor $f_{N}$, then

$$
h_{N}^{-} / h_{L}=\frac{\left(w_{N} / w_{L}\right) f_{N}^{(p-1) / 2}}{(2 \pi)^{p-1}} \prod_{\chi \in X_{N}^{-}}|L(1, \chi)| \geq\left(\frac{\sqrt{f_{N}}}{2 \pi c \log f_{N}}\right)^{p-1},
$$

which for a given $p$ is asymptotically better than the lower bound given in Corollary 21. However, even if we use the asymptotic value $c=\sqrt{295}$ (respectively, $c=\sqrt{165}$ for $p=3$ ) given in [Lou92, Theorem 1], we only infer that $h_{N}^{-}>1$ for $f \geq 2600000$ (respectively, $h_{N}^{-}>1$ for $f \geq 1300000$ and $p=3$ ), which is weaker than the result obtained in Corollary 21.

Corollary 23. Let $p \geq 3$ be an odd prime, $N_{p}=\mathbb{Q}\left(\zeta_{p}\right)$ the cyclotomic field of conductor $p$ and $h_{p}^{-}$its relative class number. Then

$$
h_{p}^{-} \geq \frac{4 \pi p}{e \sqrt{6}(p-2)}\left(\frac{3 p}{2 \pi^{4}}\right)^{(p-1) / 4} \frac{1}{\log p} .
$$

In particular, $h_{p}^{-}>2$ for $p \geq 71\left({ }^{6}\right)$.

$\left({ }^{6}\right)$ K. Uchida obtained in [Uch71b, Theorem 2] the worse bound $h_{p}^{-}>1$ for $p>2400$, and in [Uch71c, proof of Theorem, p. 576] the bound $h_{p}^{-}>1$ for $p>500$. J. M. Masley and H. L. Montgomery obtained in [MM, Section 3] the bound $h_{p}^{-}>1$ for $p>200$. See $[\mathrm{MM}]$ and [Mas75] for the solutions to the class number one and two problems for the cyclotomic fields, and [Mas76] for the determination of all the cyclotomic fields of class number $\leq 10$. 
Proof. Since $d_{N_{p}}=p^{p-2}$, the Dedekind zeta function of the quadratic subfield $L_{p}$ of $N_{p}$ has no real zero in $\left[1-2 / \log d_{N_{p}}, 1\right)$, by Corollary 11 . Now, use Remark 19, $w_{N_{p}}=2 p, Q_{N_{p}}=1$ (see [Was, Corollary 4.13]), $d_{N_{p}} / d_{N_{p}^{+}}=p^{(p-1) / 2}$ and the bound

$$
\operatorname{Res}_{s=1}\left(\zeta_{N_{p}^{+}}(s)\right) \leq\left(\pi^{2} / 6\right)^{(p-3) / 4}
$$

(see [Lou94, Lemma (ii)]).

Lemma 24 (see [Mur, Corollary 1]). Let $N$ be an abelian number field of conductor $f_{N}$ and degree $m$. Then $\varrho_{N}:=d_{N}^{1 / m} \geq \sqrt{f_{N}}$.

COROLlary $25\left({ }^{7}\right)$. Let $N$ be an imaginary abelian number field of degree $2 n \geq 4$. If $h_{N}^{-}=1$ then $f_{N} \leq 3 \cdot 10^{8}$.

Proof. If $L$ is an imaginary quadratic subfield of $N$, then $h_{L}$ divides $4 h_{N}^{-}$(see [Hor] and [Oka]), hence $h_{L}$ divides 4 . Since the class number one, two and four problems have been solved for imaginary quadratic fields, one can use [Low] or [Wat] to check that the Dedekind zeta functions of the imaginary quadratic fields of class number 1,2 or 4 have no real zero in $(0,1)$. Hence, using $(31), \sqrt{d_{N} / d_{N^{+}}} \geq d_{N}^{1 / 4}=\varrho_{N}^{n / 2} \geq f_{N}^{n / 4}$, by the previous lemma, we obtain

$$
1=h_{N}^{-} \geq \frac{2}{(2 n-1) e}\left(\frac{f_{N}}{\pi^{4}\left(\log f_{N}+\kappa_{0}\right)^{4}}\right)^{n / 4},
$$

which does imply $f_{N} \leq 3 \cdot 10^{8}$.

We are also in a position to obtain a Brauer-Siegel like result for relative class numbers:

CoROllary $26\left({ }^{8}\right)$. Let $N$ range over a set of imaginary abelian number fields. Then $\log h_{N}^{-}$is asymptotic to $\frac{1}{2} \log \left(d_{N} / d_{N^{+}}\right)$as $f_{N} \rightarrow \infty$.

$\left({ }^{7}\right)$ See $[\mathrm{Uch} 86]$ and $[$ Yam] for the solution of the class number one problem for imaginary abelian number fields, and notice that K. Uchida obtained the worse bound $h_{N}^{-}=1$ implies $f_{N} \leq 2 \cdot 10^{10}$ in [Uch86, Proposition 8].

$\left({ }^{8}\right)$ See [Lou96], and compare with [HH, Lemma 4] and [Nar, Chapter 8, Corollary 3, p. 447] which assume that $N$ ranges over a set of imaginary abelian number fields of a given degree, hence do not even apply to cyclotomic fields which are however dealt with in [Was, Theorem 4.20]. In fact, the proof of [Was, Theorem 4.20] applies to any family of imaginary abelian number fields. However, it does not yield an explicit result in the case that $N$ contains a quadratic subfield. (Compare also with [Uch71a, Theorem 2].) 
Proof. Acccording to (23), (29) and (31),

$$
\begin{gathered}
\frac{2 \sqrt{d_{N} / d_{N^{+}}}}{e \pi^{n}\left(\log f_{N}+\kappa_{0}\right)^{n}} \min \left(\frac{1}{2 n-1}, \frac{2 \pi h_{L}}{\sqrt{d_{L}} \log d_{L}}\right) \\
\leq h_{N}^{-} \leq \frac{2 w_{N} \sqrt{d_{N} / d_{N^{+}}}}{(4 \pi)^{n}}\left(\log f_{N}+\kappa_{1}\right)^{n} .
\end{gathered}
$$

We now use Siegel's theorem according to which for any $\varepsilon>0$ there exists $c_{\varepsilon}>0$ (ineffective) such that for all imaginary quadratic number fields $L$ we have $h_{L} / \sqrt{d_{L}} \geq c_{\varepsilon} d_{L}^{-\varepsilon}$ (see [Gold] for a simple proof), and the bound $f_{N}^{n / 2} \leq \sqrt{d_{N}} \leq d_{N} / d_{N^{+}}$(by Lemma 24 ), to obtain the bounds

$$
\begin{aligned}
n \log \left(\log f_{N}+\kappa_{0}\right) & =o\left(\log \left(d_{N} / d_{N^{+}}\right)\right), \\
n \log \left(\log f_{N}+\kappa_{1}\right) & =o\left(\log \left(d_{N} / d_{N^{+}}\right)\right), \\
n & =o\left(\log \left(d_{N} / d_{N^{+}}\right)\right), \\
\log w_{N} & =o\left(\log \log \left(d_{N} / d_{N^{+}}\right)\right)
\end{aligned}
$$

(for $\mathbb{Q}\left(\zeta_{w_{N}}\right) \subseteq N$ yields $\left.\sqrt{w_{N} / 2} \leq \phi\left(w_{N}\right)=\left[\mathbb{Q}\left(\zeta_{w_{N}}\right): \mathbb{Q}\right] \leq[N: \mathbb{Q}]=2 n\right)$.

\subsection{The normal case}

Lemma 27 (see [Lou03, Lemma 8(1)]). Let $N$ be a normal CM-field. Set $c=2(\sqrt{3}-1)^{2}=1.07 \ldots$ Then either

(i) there exists an imaginary quadratic subfield $L$ of $N$ such that $\zeta_{L}(s)$ and $\zeta_{N}(s)$ have a common real zero in $\left[1-c / \log d_{N}, 1\right)$, or

(ii) $\zeta_{N^{+}}(s)$ has a real zero in $\left[1-c / \log d_{N}, 1\right)$, or

(iii) $\zeta_{N}(s) \leq 0$ in $\left[1-c / \log d_{N}, 1\right)$.

Using this lemma and following the proof of Theorem 18, we obtain:

Theorem 28. Let $N$ be a normal CM-field of degree $2 n \geq 4$ and root dicriminant $\varrho_{N} \geq 50$. Set $c=2(\sqrt{3}-1)^{2}=1.07 \ldots$

(i) If $N$ contains an imaginary quadratic subfield $L$ for which $\zeta_{L}(\beta)=$ $\zeta_{N}(\beta)=0$ for some $\beta \in\left[1-c / \log d_{N}, 1\right)$, then

$$
h_{N}^{-} / h_{L} \geq \frac{2 Q_{N} w_{N} \sqrt{d_{N} / d_{N^{+}} d_{L}}}{e^{c / 2}\left(\frac{\pi e}{n-1} \log d_{N^{+}}\right)^{n-1} \log ^{2} d_{L}},
$$

which implies

$$
h_{N}^{-} \geq \frac{c Q_{N} w_{N} \sqrt{d_{N} / d_{N^{+}}}}{2 \pi e^{c / 2}\left(\frac{\pi e}{n-1} \log d_{N^{+}}\right)^{n-1} \log d_{N}} \cdot \frac{4 \pi n h_{L}}{c \sqrt{d_{L}} \log d_{L}} .
$$


(ii) Otherwise,

$$
h_{N}^{-} \geq \frac{c Q_{N} w_{N} \sqrt{d_{N} / d_{N^{+}}}}{2 \pi e^{c / 2}\left(\frac{\pi e}{n-1} \log d_{N^{+}}\right)^{n-1} \log d_{N}} .
$$

Proof. (i) Since $\zeta_{L}(\beta)=\zeta_{N}(\beta)=0$, we have

$$
\operatorname{Res}_{s=1}\left(\zeta_{N}(s)\right) \geq \frac{1}{2}(1-\beta) d_{N}^{(\beta-1) / 2} \geq \frac{1-\beta}{2 e^{c / 2}}
$$

by (25), and

$$
\operatorname{Res}_{s=1}\left(\zeta_{L}(s)\right) \leq \frac{1-\beta}{8} \log ^{2} d_{L}
$$

by Theorem 1 . Moreover,

$$
\operatorname{Res}_{s=1}\left(\zeta_{N^{+}}(s)\right) \leq\left(\frac{e \log d_{N^{+}}}{2(n-1)}\right)^{n-1}
$$

by Theorem 14. Hence, using (21) for $N$ and $L$ and noticing that $Q_{L}=1$, $w_{L}=2$ and $\operatorname{Res}_{s=1}\left(\zeta_{L^{+}}(s)\right)=\operatorname{Res}_{s=1}(\zeta(s))=1$, we obtain $(36)$.

(ii)(a) Assume that $\zeta_{N^{+}}(s)$ has a real zero $\beta \in\left[1-c / \log d_{N}, 1\right)$. Then

$$
\operatorname{Res}_{s=1}\left(\zeta_{N}(s)\right) \geq \frac{1}{2}(1-\beta) d_{N}^{(\beta-1) / 2} \geq \frac{1-\beta}{2 e^{c / 2}}
$$

by (25), and

$$
\operatorname{Res}_{s=1}\left(\zeta_{N^{+}}(s)\right) \leq \frac{1-\beta}{4(n-1)}\left(\frac{e \log d_{N^{+}}}{2(n-1)}\right)^{n-1} \log d_{N}
$$

by Theorem 14 (for $((n-1) / n)^{n} \leq 1 / e$ and $\left.d_{N^{+}} \leq \sqrt{d_{N}}\right)$. Hence, using (21), we infer that (38) is valid.

(b) It remains to consider the case that $\zeta_{N}(s) \leq 0$ for $s \in\left[1-c / \log d_{N}, 1\right)$. Then $\zeta_{N}\left(1-2 / \log d_{N}\right) \leq 0$. Hence,

$$
\operatorname{Res}_{s=1}\left(\zeta_{N}(s)\right) \geq \frac{2}{e \log d_{N}}
$$

by (26). Moreover,

$$
\operatorname{Res}_{s=1}\left(\zeta_{N^{+}}(s)\right) \leq\left(\frac{e \log d_{N^{+}}}{2(n-1)}\right)^{n-1}
$$

by Theorem 14. Hence, using (21), we conclude that (38) is here also valid.

Corollary 29 (cf. [Uch71a, Thm. 1]). Let $N$ range over a set of normal $C M$-fields such that $\varrho_{N} \rightarrow \infty$. Then $\log h_{N}^{-}$is asymptotic to $\frac{1}{2} \log \left(d_{N} / d_{N^{+}}\right)$.

\subsection{The nonnormal case}

Lemma 30. Let $N$ denote the normal closure of a CM-field $K$ of degree $2 n$. 
(i) $d_{N}$ divides $d_{K}^{n[N: K]}$ and $[N: K]$ divides $2^{n-1}(n-1)$ !.

(ii) (see [Lou03, Lemma 8(3)]) Set $c=2(\sqrt{2}-1)^{2}=0.343 \ldots$ Then either

(a) there exists an imaginary quadratic subfield $L$ of $K$ such that $\zeta_{L}(s)$ and $\zeta_{K}(s)$ have a common real zero in $\left[1-c / \log d_{N}, 1\right)$, or

(b) $\zeta_{K^{+}}(s)$ has a real zero in $\left[1-c / \log d_{N}, 1\right)$, or

(c) $\zeta_{K}(s) \leq 0$ for $s \in\left[1-c / \log d_{N}, 1\right)$.

Proof. Let $K_{1}^{+}, \ldots, K_{n}^{+}$be the real embeddings of $K^{+}$, and $\alpha_{1}, \ldots, \alpha_{n}$ be the conjugates of $\alpha \in K^{+}$such that $K=K^{+}(\sqrt{\alpha})$. Since we have $N=$ $K_{1}^{+}\left(\sqrt{\alpha_{1}}\right) \cdots K_{n}^{+}\left(\sqrt{\alpha_{n}}\right)$ and $d_{K_{i}^{+}\left(\sqrt{\alpha_{i}}\right)}=d_{K}$, [Sta, Lemma 7] yields the first assertion of (i). For the second one, notice that $N=\widehat{K}^{+}\left(\sqrt{\alpha_{1}}, \ldots, \sqrt{\alpha_{n}}\right)$, where $\widehat{K}^{+}$is the normal closure of $K$.

Using this lemma and (27), and following the proof of Theorem 28, we obtain:

THEOREM 31. Let $N$ denote the normal closure of a nonnormal CM-field $K$ of degree $2 n \geq 4$ and root discriminant $\varrho_{N} \geq 2683$. Set $c=2(\sqrt{2}-1)^{2}$ $=0.343 \ldots$ and $\alpha=\left(\log d_{K}\right) / \log d_{N} \in\left[1 / 2^{n-1} n !, 1 / 2\right]$.

(i) If $K$ contains an imaginary quadratic subfield $L$ for which $\zeta_{L}(\beta)=$ $\zeta_{K}(\beta)=0$ for some $\beta \in\left[1-c / \log d_{N}, 1\right)$, then

$$
h_{K}^{-} / h_{L} \geq \frac{4 Q_{K} w_{K} \sqrt{d_{K} / d_{K^{+}} d_{L}}}{e^{c \alpha / 2}\left(\frac{\pi e}{n-1} \log d_{K^{+}}\right)^{n-1} \log ^{2} d_{L}},
$$

which implies

$$
h_{K}^{-} \geq \frac{c \alpha Q_{K} w_{K} \sqrt{d_{K} / d_{K^{+}}}}{2 \pi e^{c \alpha / 2}\left(\frac{\pi e}{n-1} \log d_{K^{+}}\right)^{n-1} \log d_{K}} \cdot \frac{8 \pi n h_{L}}{c \alpha \sqrt{d_{L}} \log d_{L}} .
$$

(ii) Otherwise,

$$
h_{K}^{-} \geq \frac{c \alpha Q_{K} w_{K} \sqrt{d_{K} / d_{K^{+}}}}{2 \pi e^{c \alpha / 2}\left(\frac{\pi e}{n-1} \log d_{K^{+}}\right)^{n-1} \log d_{K}} .
$$

COROLlary 32. Let $K$ range over a set of nonnormal CM-fields of a given degree. Then $\log h_{K}^{-}$is asymptotic to $\frac{1}{2} \log \left(d_{K} / d_{K^{+}}\right)$.

\section{References}

[AD] F. Amoroso and R. Dvornicich, Lower bounds for the height and size of the ideal class group in CM-fields, Monatsh. Math. 138 (2003), 85-94. 
[Bes] S. Bessassi, Bounds for the degrees of CM-fields of class number one, Acta Arith. 106 (2003), 213-245.

[BL] G. Boutteaux and S. Louboutin, The class number one problem for some nonnormal sextic CM-fields, in: Analytic Number Theory (Beijing/Kyoto, 1999), Kluwer, 2002, 27-37.

[CK98] K.-Y. Chang and S.-H. Kwon, Class number problem for imaginary cyclic number fields, J. Number Theory 73 (1998), 318-338.

[CK00a] - - C Class numbers of imaginary abelian number fields, Proc. Amer. Math. Soc. 128 (2000), 2517-2528.

[CK00b] - - - The imaginary abelian number fields with class numbers equal to their genus class numbers, in: Colloque International de Théorie des Nombres (Talence, 1999), J. Théor. Nombres Bordeaux 12 (2000), 349-365.

[Dav] H. Davenport, Multiplicative Number Theory, 3rd ed., revised and with a preface by H. L. Montgomery, Grad. Texts in Math. 74, Springer, New York, 2000.

[Gold] D. M. Goldfeld, A simple proof of Siegel's theorem, Proc. Nat. Acad. Sci. USA 71 (1974), 1055.

[HHRW] K. Hardy, R. Hudson, D. Richman and K. S. Williams, Determination of all imaginary cyclic quartic fields with class number 2, Trans. Amer. Math. Soc. 311 (1989), 1-55.

[Has] H. Hasse, Über die Klassenzahl abelscher Zahlkörper, reprint of the 1952 edition, with an introduction by J. Martinet, Akademie-Verlag, Berlin, 1985.

[Hor] K. Horie, On a ratio between relative class numbers, Math. Z. 211 (1992), $505-521$.

[HH] K. Horie and M. Horie, CM-fields and exponents of their ideal class groups, Acta Arith. 55 (1990), 157-170.

[Kob] N. Koblitz, Introduction to Elliptic Curves and Modular Forms, 2nd ed., Grad. Texts in Math. 97, Springer, New York, 1993.

[Lem] F. Lemmermeyer, Ideal class groups of cyclotomic number fields. I, Acta Arith. 72 (1995), 347-359.

[LPP] H. W. Lenstra, J. Pila and C. Pomerance, A hyperelliptic smoothness test, II, Proc. London Math. Soc. 84 (2002), 105-146.

[Lou92] S. Louboutin, Minoration au point 1 des fonctions $L$ et détermination des corps sextiques abéliens totalement imaginaires principaux, Acta Arith. 62 (1992), 109-124.

[Lou94] - Lower bounds for relative class numbers of CM-fields, Proc. Amer. Math. Soc. 120 (1994), 425-434.

[Lou95] - Determination of all nonquadratic imaginary cyclic number fields of 2power degrees with ideal class groups of exponents $\leq 2$, Math. Comp. 64 (1995), $323-340$.

[Lou96] -, A finiteness theorem for imaginary abelian number fields, Manuscripta Math. 91 (1996), 343-352.

[Lou97] -, CM-fields with cyclic ideal class groups of 2-power orders, J. Number Theory 67 (1997), 1-10.

[Lou98a] - , The imaginary cyclic sextic fields with class numbers equal to their genus class numbers, Colloq. Math. 75 (1998), 205-212.

[Lou98b] -, Majorations explicites du résidu au point 1 des fonctions zêta des corps de nombres, J. Math. Soc. Japan 50 (1998), 57-69. 
[Lou99] S. Louboutin, The nonquadratic imaginary cyclic fields of 2-power degrees with class numbers equal to their genus class numbers, Proc. Amer. Math. Soc. 127 (1999), 355-361.

[Lou00] - Explicit bounds for residues of Dedekind zeta functions, values of L-functions at $s=1$, and relative class numbers, J. Number Theory 85 (2000), 263-282.

[Lou01] - Explicit upper bounds for residues of Dedekind zeta functions and values of $L$-functions at $s=1$, and explicit lower bounds for relative class numbers of CM-fields, Canad. J. Math. 53 (2001), 1194-1222.

[Lou03] - Explicit lower bounds for residues at $s=1$ of Dedekind zeta functions and relative class numbers of CM-fields, Trans. Amer. Math. Soc. 355 (2003), 3079-3098.

[Lou04] —, Explicit upper bounds for $|L(1, \chi)|$ for primitive characters $\chi$, Quart. J. Math. 55 (2004), 57-68.

[LO] S. Louboutin and R. Okazaki, Exponents of the ideal class groups of CM number fields, Math. Z. 243 (2003), 155-159.

[LOO] S. Louboutin, R. Okazaki and M. Olivier, The class-number one problem for some non-abelian normal CM-fields, Trans. Amer. Math. Soc. 349 (1997), $3657-3678$.

[Low] M. E. Low, Real zeros of the Dedekind zeta function of an imaginary quadratic field, Acta Arith. 14 (1967/1968), 117-140.

[Mas75] J. M. Masley, Solution of the class number two problem for cyclotomic fields, Invent. Math. 28 (1975), 243-244.

[Mas76] - Solution of small class number problems for cyclotomic fields, Compositio Math. 33 (1976), 179-186.

[MM] J. M. Masley and H. L. Montgomery, Cyclotomic fields with unique factorization, J. Reine Angew. Math. 286/287 (1976), 248-256.

[Mur] M. R. Murty, An analogue of Artin's conjecture for abelian extensions, J. Number Theory 18 (1984), 241-248.

[Nar] W. Narkiewicz, Elementary and Analytic Theory of Algebraic Numbers, 2nd ed., Springer, Berlin, 1990.

[Oka] R. Okazaki, Inclusion of CM-fields and divisibility of relative class numbers, Acta Arith. 92 (2000), 319-338.

[Pin] J. Pintz, Elementary methods in the theory of L-functions, II. On the greatest real zero of a real $L$-function, ibid. 31 (1976), 273-289.

[Rad] H. Rademacher, Topics in Analytic Number Theory, Springer, 1973.

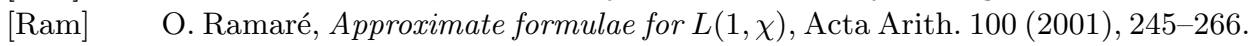

[Sta] H. M. Stark, Some effective cases of the Brauer-Siegel theorem, Invent. Math. 23 (1974), 135-152.

[Uch71a] K. Uchida, Class numbers of imaginary abelian number fields. I, Tôhoku Math. J. 23 (1971), 97-104.

[Uch71b] -, Class numbers of imaginary abelian number fields. II, ibid. 23 (1971), 335348.

[Uch71c] -, Class numbers of imaginary abelian number fields. III, ibid. 23 (1971), $573-580$.

[Uch72] - Imaginary abelian number fields with class number one, ibid. 24 (1972), 487-499.

[Uch86] - Imaginary abelian number fields of degrees $2^{m}$ with class number one, in: Proc. Internat. Conf. on Class Numbers and Fundamental Units of Algebraic Number Fields (Katata, 1986), Nagoya Univ., Nagoya, 1986, 151-170. 
[Was] L. C. Washington, Introduction to Cyclotomic Fields, 2nd ed., Grad. Texts in Math. 83, Springer, New York, 1997.

[Wat] M. Watkins, Real zeros of real odd Dirichlet L-functions, Math. Comp. 73 (2004), 415-423.

[Yam] K. Yamamura, The determination of the imaginary abelian number fields with class number one, ibid. 62 (1994), 899-921.

Institut de Mathématiques de Luminy, UMR 6206

163, avenue de Luminy, Case 907

13288 Marseille Cedex 9, France

E-mail: loubouti@iml.univ-mrs.fr

Received on 21.2.2005

and in revised form on 22.6.2005 